\title{
Aerodynamic optimization of through-flow design model of a high by-pass transonic aero-engine fan using genetic algorithm
}

Proc IMechE Part A:

$J$ Power and Energy

$0(0) \mathrm{I}-14$

(C) IMechE 2017

Reprints and permissions:

sagepub.co.uk/journalsPermissions.nav DOI: 10.1 I77/09576509|7730466

journals.sagepub.com/home/pia

(SSAGE

\author{
Orçun Kor', Sercan Acarer ${ }^{2}$ and Ünver Özkol ${ }^{3}$
}

\begin{abstract}
This study deals with aerodynamic optimization of a high by-pass transonic aero-engine fan module in a through-flow inverse design model at cruise condition. To the authors' best knowledge, although the literature contains through-flow optimization of the simplified cases of compressors and turbines, an optimization study targeting the more elaborate case of combined transonic fan and splitter through-flow model is not considered in the literature. Such a through-flow optimization of a transonic fan, combined with bypass and core streams separated by an aerodynamically shaped flow splitter, possesses significant challenges to any optimizer, due to highly non-linear nature of the problem and the high number of constraints, including the fulfillment of the targeted bypass ratio. It is the aim of this study to consider this previously untouched area in detail and therefore present a more sophisticated and accurate optimization environment for actual bypass fan systems. An in-house optimization code using genetic algorithm is coupled with a previously developed in-house through-flow solver which is using a streamline curvature technique and a set of in-house calibrated empirical models for incidence, deviation, loss and blockage. As the through-flow models are the backbone of turbomachinery design, and great majority of design decisions are taken in this phase, such a study is assessed to result in significant guidelines to the gas turbine community.
\end{abstract}

\section{Keywords}

Fans, turbomachinery aerodynamics, optimization, through-flow, genetic algorithm, air-breathing engines, axial flow compressors, compressor aerodynamics, axial fans

Date received: 15 January 2017; accepted: 4 August 2017

\section{Introduction}

Two-dimensional through-flow design analyses of turbomachinery aerothermodynamics in aero-engines, mostly conducted by streamline curvature (SLC) methods, has upmost importance due to the fact that majority $(\sim 80 \%)$ of the design is fixed in the pre-three-dimensional (mean-line, through-flow and blade-to-blade) design steps in the today's state-ofthe-art. ${ }^{1}$ Once the aerodynamic objectives, for example maximizing efficiency, maximizing the uniformity of total pressure distribution, etc., are predominantly optimized in the through-flow design, one will spend much less time in the computationally expensive three-dimensional aerodynamic design phase. In addition to the advantages of the method mentioned above, accuracy and effectiveness of the throughflow methods make them the backbone of turbomachinery aerodynamic design. Therefore, for a robust final design, a well-tailored through-flow design should be provided.

The computational model lying behind the through-flow solver (see The utilized through-flow method section for details) is not only highly non-linear, but also discrete, discontinuous and nondifferentiable, containing many local peaks. Furthermore, the multi-variable nature of the

\footnotetext{
'R\&D Department, TUSAŞ Engine Industries, Eskisehir, Turkey

${ }^{2}$ Department of Mechanical Engineering, İzmir Katip Çelebi University, Izmir, Turkey

${ }^{3}$ Department of Mechanical Engineering, İzmir Institute of Technology, Izmir, Turkey

Corresponding author:

Orçun Kor, Tusas, Engine Industries, Esentepe Mh. Cevreyolu Blv. No: 356, 26003 Tepebasi, Eskisehir, Turkey.

Email: orcunkor@gmail.com
} 
problem introduces additional complexities in case an optimum solution is targeted. Designer would face several disadvantages if the optimization task is done manually, since meeting multiple requirements simultaneously is not only vulnerable to humansourced errors, but also it does not guarantee a complete scan of the design space. One therefore has to conduct an automatic optimization procedure that is discussed in following paragraphs.

The literature about turbomachinery optimization reports the use of both gradient-based and stochastic techniques. ${ }^{2}$ Each technique has its own advantages depending on the character of the problem and the aim of the designer; therefore it cannot be stated that one is superior to others. In case a reliable and feasible initial guess is provided, gradient-based algorithms will perform in a fast and accurate fashion and find the local optimum with ease. These methods are based on pure mathematical operations; therefore, their stability will highly be dependent on objective/ constraint functions' differentiability. On the other hand, the design variables in some problems are solely allowed to take integer values, such as the number of blades in a rotor disc, where differentiation problem will occur. These issues are reported to be main limitations in gradient-based optimizers. ${ }^{3,4}$ Some novel methods benefiting the service of adjoint operator and control theory ${ }^{5,6}$ also use gradient information. These methods search the optimum based on the sensitivity of given functions which are dependent on preselected design variables. They are powerful, accurate and low cost in terms of computational time, and reported to be robust and able to outwit the stability problems. On the other hand, as observed in other gradient based optimizers, the method is applicable only if it is known that the objective function does not have many local minima, otherwise, it generally converges to the nearest local minimum. ${ }^{4}$ In addition, the complexity and programming difficulties of the adjoint method (in particular, discrete adjoint method) make it less preferable, despite of its many advantages in search for optimum. ${ }^{7}$ The literature points successful efforts on the use of gradient-based methods as a part of through-flow optimization of compressors or turbines. One example is the study by Massardo, ${ }^{8,9}$ where the through-flow problem is modelled by a non-linear program and subjected to a gradient search for the purpose of decreasing losses and compressor weight, and increasing stall margin. Burguburu et al. ${ }^{10}$ also utilized a gradient-based approach with the motivation of minimizing losses by perturbing the turbine blade geometry. The study shows that significant improvements on objective functions are obtained, fulfilling the constraint requirements.

Stochastic techniques, on the other hand, are suggested to be powerful in the search for global optimum. Genetic algorithm, one of most commonly used stochastic optimizers, is reported as robust and accurate, and suitable to be used in turbomachinery problems. ${ }^{11-13}$ Piérret may be the first researcher, who unravels the performance of genetic algorithms on a multi-objective turbomachinery problem, obtaining significant improvements of Mach number distribution on a turbine blade, leading to reduced losses on the order of $4 \%{ }^{14,15}$ Similarly, extensive number of studies has been performed about turbomachinery optimization, especially on blade tailoring on cascade plane and also in three-dimensional sense. ${ }^{2,16-19}$ However, reports on through-flow design optimization not only by genetic algorithm, but also by other stochastic methods are quite limited, even in the simplified case of single-stream compressor and turbines. The study of Petrovic et al. ${ }^{20}$ presents an application of genetic algorithm (hybridized with gradient method) on through-flow optimization of a turbine, where maximum efficiency is provided. In a similar through-flow optimization study, Park et al. ${ }^{21}$ obtained $0.5-1.5 \%$ increase in efficiency of a threestage low pressure compressor by perturbing booster hub and shroud geometries using hybrid genetic algorithm. Joly et al. ${ }^{22}$ recently use differential evolution for optimizing a highly loaded fan with a pressure ratio of about 2, including the through-flow phase, where they obtained maximum efficiency, minimum tip radius of the machine and lowest value of relative flow turning over the spans of supercritical profiles; however, the geometry considered is single stream fan where the splitter is not included.

In this study, an almost untouched but highly critical area is considered. Instead of the simplified cases of single stream compressors and turbines, this study deals with multi-objective optimization of the unified system of high-speed transonic fan, flow splitter and two downstream channels with bypass outlet and core inlet guide vanes (OGV and IGV) by using an inhouse novel through-flow inverse design model with in-house calibrated empirical models for incidence, deviation, loss and blockage, previously developed and validated by Acarer and Özkol. ${ }^{23,24}$ As opposed to investigating a sole fan without true description of the splitter and two downstream ducts, as commonly done in prior studies, optimization of such a combined system possesses additional challenge due to highly non-linear nature of the problem and the existence of higher number of realistic constraints such as fulfilment of the targeted bypass ratio. The optimization code developed by Kor $^{25}$ using genetic algorithm, validated against several generic optimization test cases, is taken into service for this sake. In this regard, a more complete design model for bypass fans is expected to be developed. As consistent with typical inverse through-flow design phase studies, which are used to determine preliminary geometry for the design point, the 'level cruise' condition is optimized in this study. For high bypass civil turbofans, majority of flight time is spent during level cruise and efficiency is the most important parameter. Other 
critical operating points, such as take-off, are not considered during this design stage, and instead classified as a part of later off-design performance study. The following sections describe the through-flow and optimization programs, optimization goals and strategy and the results.

\section{The utilized through-flow method}

The utilized through-flow methodology is described and validated in detail in Acarer and Özkol ${ }^{23,24}$ and only a brief summary of the study is presented here. The SLC method is used to solve the through-flow problem of a bypass fan system. The momentum equation given in equation (1) is solved along each quasi-orthogonal, which are approximately orthogonal to streamlines

$$
\begin{aligned}
& V_{m} \frac{\partial V_{m}}{\partial q}=\frac{\partial H}{\partial q}-T \frac{\partial s}{\partial q}-\frac{V_{\theta}}{r} \frac{\partial\left(r V_{\theta}\right)}{\partial q} \\
& +V_{m} \sin \varepsilon \frac{\partial V_{m}}{\partial m}+K_{m} V_{m}^{2} \cos \varepsilon-F_{q}
\end{aligned}
$$

Stream-wise momentum equation is reduced to conservation of stagnation enthalpy, pressure and angular momentum, within duct regions, between successive quasi-orthogonals. For solution within bladed regions, the turbomachinery energy equation and cascade incidence, deviation and total pressure loss correlations must be applied. ${ }^{23,26}$ The blade force term ' $F_{q}$ ' is neglected in this study since no lean is considered. However, these correlations are only initial estimates and actual incidence, deviation and loss are later determined through future iterations with blade-to-blade and/or three-dimensional calculations. The streamline positions are being updated on quasiorthogonals' using continuity until convergence with the streamline positions is achieved.

The utilized code implements inverse mode of solution, where tangential velocity $\left(V_{\theta}\right)$ distributions at the trailing edge of each blade row (similarly $H$ or indirectly total pressure) are specified and the corresponding flow field is sought. The blade sections are adjusted to operate under minimum-loss incidence conditions. This implementation is called also 'design mode' in some of the literature.

The solution of bypass fan requires the flow to split into two main streams by-pass flow and core engine flow (Figures 1 and 2). This scheme is already developed and implemented in the through-flow methodology ${ }^{23}$ as an inverse-design-oriented modification of the conventional method of Novak. ${ }^{27}$ The methodology is based on the fact that upstream propagation of any downstream obstacle in a streamline-curvature model is taken into account geometrically, that is by streamline slope $(\phi)$ and curvature $\left(K_{m}\right)$ terms of equation (1), as well as the $\delta V_{m} / \delta m$ term. Therefore, a solution can be obtained by iterative solution of subdomains, only if the complete network

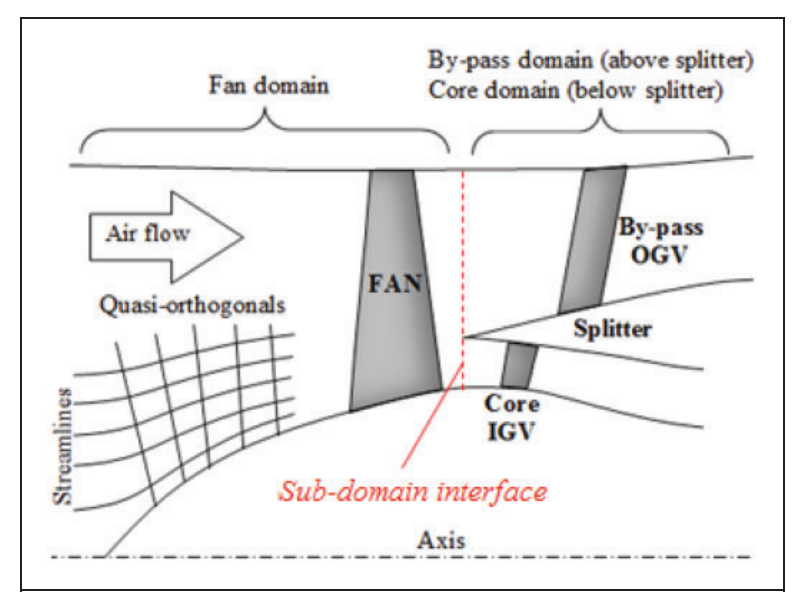

Figure I. Streamline curvature method. ${ }^{23}$ IGV: inlet guide vane; OGV: outlet guide vane.

of streamlines is used to calculate local streamline slope and curvature terms. Subdomains, as seen in Figures 1 and 2(b), consist of fan (upstream of the splitter), bypass (bypassing stream above the splitter) and core (core engine stream below the splitter). The exact outlet of the fan domain is a quasi-orthogonal itself and coincides with the splitter leading edge to form an interface with by-pass and core domains. Although the split-flow method is very similar with the original method of Novak, ${ }^{27}$ the by-pass ratio comes out as a result of calculations such that the flow always approaches aligned to the already existing splitter contour. In the conventional method of Novak, the bypass ratio is an input parameter; therefore the flow can approach to the splitter with a large angle of attack to satisfy the desired bypass ratio, which is disadvantageous in terms of solution convergence. Apart from better convergence, the utilized method is assessed to be advantageous during inverse design as flow alignment to the splitter leading edge is guaranteed.

\section{Empiricial models}

List of the utilized models, together with the references, are listed in Table 1. The summary of those correlations, originally presented in the authors' prior study, ${ }^{23}$ are re-touched in the following text.

In the design mode, only the flow angles at the inlet and outlet are known from the through-flow calculation. However, the loss models require inlet and outlet metal angles. Therefore, the procedure starts with the calculation of flow incidence and deviation to obtain metal angles, which correspond to minimum-loss operation. Before obtaining the final (Machcorrected, etc.) incidence and deviation, initially, the reference (low-speed) minimum - loss incidence $\left(i^{*}\right)$ and the corresponding deviation $\left(\delta^{*}\right)$ are obtained from the well-known Lieblein models. ${ }^{28,29}$ Once the reference minimum-loss incidence angle is determined, the reference stall and choke angular ranges 


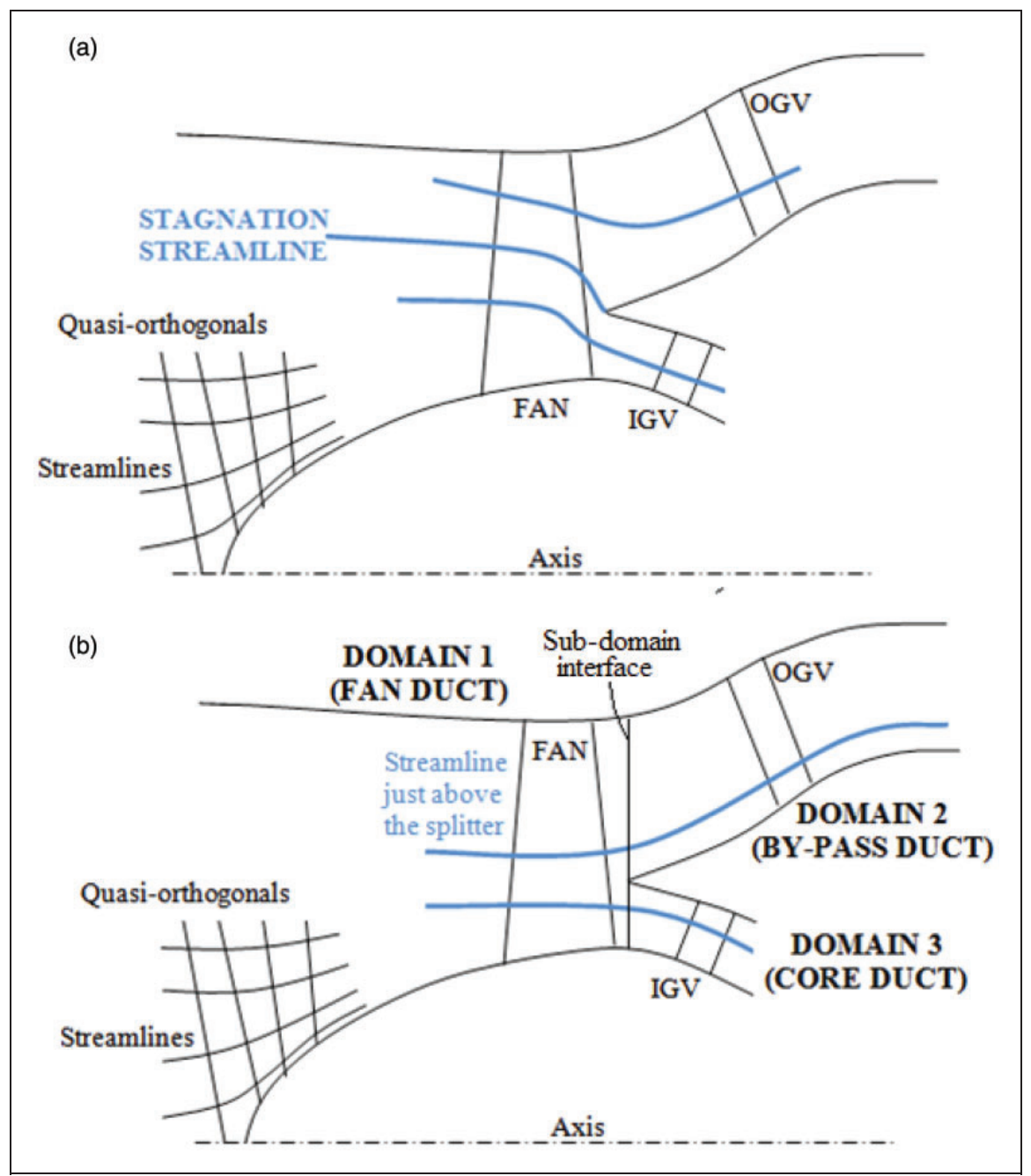

Figure 2. Conventional ${ }^{27}$ (a) and modified ${ }^{23}$ (b) by-pass flow schemes (taken from Acarer and Özkol ${ }^{23}$ ). IGV: inlet guide vane; OGV: outlet guide vane.

are required for further compressibility corrections. As described in the original study, ${ }^{23}$ the angular range from reference minimum-loss incidence to reference choke incidence $\left(i_{c}\right)$, denoted as $R_{c}$, can be estimated from Aungier ${ }^{26}$

$$
\mathrm{i}_{\mathrm{c}}-\mathrm{i}=-\mathrm{R}_{\mathrm{c}}=-\left(10-\theta \frac{\left(\mathrm{K}_{1}-40\right)}{450}\right)\left(0.5+5 \frac{\mathrm{th}}{\mathrm{c}}\right)
$$

where $t h / c$ is the ratio of maximum profile thickness to the chord length, $\theta$ is camber and $K_{1}$ is inlet metal angle. Similarly, the reference angular range from the reference minimum-loss incidence to the reference stall incidence $\left(i_{s}\right)$, denoted as $R_{s}$, can be estimated by Aungier ${ }^{26}$

$$
i_{s}-i^{*}=-R_{s}=1.5\left(10+\theta \frac{\left(55-K_{1}\right)}{150}\right)\left(0.5+5 \frac{t h}{c}\right)
$$

As expected, these ranges get smaller significantly as the inlet Mach number, and therefore compressibility, increases. Aungier ${ }^{26}$ suggests below corrections for compressibility effects

$$
\begin{aligned}
& i_{c(\text { compressible })}=i^{*}-R_{c} /\left[1+0.5\left(M_{1}^{\prime}\right)^{3}\right] \\
& i_{s(\text { compressible })}=i^{*}+R_{s} /\left[1+0.5\left(K_{s h} M_{1}^{\prime}\right)^{3}\right]
\end{aligned}
$$

Also, $i_{c}$ must be higher than the real choke incidence, which will result in $2 \%$ more mass flow rate than the choke mass flow of the section (according to Aungier's practice ${ }^{26}$ ). This limit results

$$
i_{c(\text { compressible })}=\beta_{1, \text { choke }}-K_{1}+1^{\circ}
$$

where $\beta_{1, \text { choke }}$ is the flow angle at which passage chokes at the throat. It may be estimated from

$$
\rho_{1} W_{1} t \cos \beta_{1, \text { choke }}=o \rho_{\text {sonic }} W_{\text {sonic }}
$$

where ' $O$ ' is the throat opening of investigated section. Unless directly obtained from the geometry, which is highly advised for direct analysis mode but cannot be 
Table I. Employed empirical models. ${ }^{23}$

\begin{tabular}{|c|c|}
\hline $\begin{array}{l}\text { Reference minimum-loss } \\
\text { incidence }\end{array}$ & Lieblein $^{28}$ or NASA SP- $36^{29}$ \\
\hline $\begin{array}{l}\text { Reference stall-choke } \\
\text { incidence range }\end{array}$ & Kleppler $^{30}$ \\
\hline Optimum stagger & Aungier ${ }^{26}$ \\
\hline Throat & Aungier ${ }^{26}$ \\
\hline $\begin{array}{l}\text { Mach corrected stall- } \\
\text { choke range }\end{array}$ & Aungier ${ }^{26}$ \\
\hline $\begin{array}{l}\text { Mach corrected min. loss } \\
\text { incidence }\end{array}$ & Aungier $^{26}$ \\
\hline Reference deviation & Lieblein $^{28}$ or NASA SP-36 $6^{29}$ \\
\hline Deviation correction & Boyer $^{31}$ \\
\hline Equivalent diffusion factor & Koch and Smith ${ }^{32}$ \\
\hline $\begin{array}{l}\text { Friction (profile }+ \text { second- } \\
\text { ary) loss coefficient }\end{array}$ & $\begin{array}{l}\text { Aungier }{ }^{26} \text { (based on NASA } \\
\quad \text { SP- } 36^{29} \text { ) }\end{array}$ \\
\hline $\begin{array}{l}\text { Transonic shock loss } \\
\text { coefficient }\end{array}$ & Aungier ${ }^{26}$ \\
\hline $\begin{array}{l}\text { Supersonic shock loss } \\
\text { coefficient }\end{array}$ & $\begin{array}{l}\text { Miller et al. }{ }^{33} \text { and } \\
\text { Wennerstrom }\end{array}$ \\
\hline End-wall blockage & Pachidis $^{35}$ \\
\hline Off-design loss & Çetin et al. $^{36}$ \\
\hline Off-design deviation & Creveling $^{37}$ \\
\hline
\end{tabular}

done during the inverse blade design as the blades are not known, empirical formulas, such as the ones given by Aungier ${ }^{26}$ have to be to be employed to roughly estimate the throat-to-pitch ratio. One should keep in mind that throat estimation correlations may not be reliable and may give rise to significant errors, especially for high speed cases. Therefore, they should only be used in the very beginning of the design phase with caution and may need to be calibrated (if required) for specific cases. The baseline correlation given by Aungier ${ }^{26}$ is implemented to the methodology

$$
\frac{o}{t}=\left[\left(1-\frac{t h \sqrt{\sigma}}{c}\right) \cos \Psi\right]^{\sqrt{\sigma}}
$$

where $\Psi$ is modified stagger parameter defined as

$$
\Psi=\gamma\left[1-\frac{1}{20}\left(\frac{\tan \left(\frac{\theta}{4}\right) a}{0.05515} \frac{a}{c}\right)^{1.5}\right]+5\left(\frac{\tan \left(\frac{\theta}{4}\right)}{0.05515} \frac{a}{c}\right)^{1.5}-2
$$

where $a / c$ is the chord-wise location of maximum camber point in a parabolic camberline shape. ${ }^{26}$ The corresponding stagger can be assumed from

$$
\gamma=K_{1} \tan ^{-1}\left[4 \frac{b}{c} /\left(4 \frac{a}{c}-1\right)\right]
$$

In this equation, $b$ is the maximum distance between the camberline and the chordline, which is at the position corresponding to $a / c . b / c$ ratio is given by $\mathrm{Novak}^{27}$

$$
\frac{b}{c}=\left\{\sqrt{1+(4 \tan \theta)^{2}\left[\frac{a}{c}-\left(\frac{a}{c}\right)^{2}-\frac{3}{16}\right]}-1\right\} / \tan \theta
$$

Once those parameters are calculated, the compressibility-corrected minimum loss incidence at a given section is obtained by Aungier ${ }^{26}$

$$
i_{m}=\frac{i_{c}+\left(i_{s}-i_{c}\right) R_{c}}{\left(R_{c}+R_{s}\right)}
$$

For the deviation $\left(\delta^{*}\right)$, a correction is also applied for meridional acceleration or deceleration, which has a significant impact, as described by Boyer ${ }^{31}$

$$
\delta_{V_{m}}=10\left(1-\frac{V_{m, 2}}{V_{m, 1}}\right)
$$

Deviation is not corrected for inlet relative Mach number effects due to having a minor effect. ${ }^{31}$

The total pressure loss coefficient is composed of friction (profile + secondary) and shock loss coefficients. Similar to the incidence, the reference loss coefficient is obtained initially, and then several corrections are included. The reference (low-speed) friction loss coefficient is based on NASA SP-36 loss correlations ${ }^{29}$

$$
\omega^{*}=\frac{2 \sigma C_{1}\left[C_{2}+3.1\left(D_{e q}-1\right)^{2}+0.4\left(D_{e q}-1\right)^{8}\right]}{\cos \beta_{2}}\left(\frac{W_{2}}{W_{1}}\right)^{2}
$$

where $C_{1}$ and $C_{2}$ are calibration coefficients. $C_{1}$ is taken as $0.0073,{ }^{26}$ while $C_{2}$ considers the secondary losses. Aungier ${ }^{26}$ suggests below formula for the secondary loss

$$
C_{2}=1+\frac{t_{\text {average }}}{c} \cos \beta_{2}
$$

The friction loss coefficient, defined by equation (14), is corrected for Mach number effects as

$$
\omega_{\text {friction }}=\omega^{*}\left[1+\frac{\left(i_{m}-i^{*}\right)^{2}}{R_{s}^{2}}\right]
$$

Shock losses are considered in two ways (1) for transonic profiles, where inlet Mach number is subsonic but there are supersonic pockets within the blade passage; (2) the supersonic profiles, where the inlet relative Mach number is supersonic. For transonic sections, below relation is used ${ }^{26}$

$$
\omega_{\text {shock(transonic })}=K_{\text {sh }}\left[\frac{\left(M_{1}^{\prime} / M_{\text {critical }}-1\right) W_{\text {sonic }}}{W_{1}}\right]^{2}
$$


For supersonic-inlet sections, MLH model ${ }^{33}$ is used as a baseline to calculate shock losses

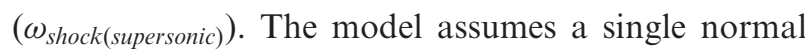
shock at upstream Mach number calculated as the geometric average $^{26}$ of inlet relative Mach number and accelerated suction surface Mach number. Wennerstrom $^{34}$ model is utilized to consider 3D obliqueness to modify the average Mach number.

The through-flow calculation also requires effective passage area to consider boundary layer growth. For this purpose, the method utilized by Pachidis ${ }^{35}$ is employed. The methodology also contains off-design correlations given by Çetin et al. ${ }^{36}$ and Creveling, ${ }^{37}$ although not used in this study since only design point is considered here.

Several calibrations to the enlisted models had been made in the prior study ${ }^{23}$ based on peak efficiency operating points of NASA 2-stage $\operatorname{fan}^{38}$ and a custom designed fan stage. The performance was then tested in GE-NASA bypass fan ${ }^{39}$ with success. Such a calibration to specific cases is required since the empirical models are oversimplified, not universal and they are case-specific, although they are developed based on extensive compressor cascade test data over many years. This is because losses in real turbomachinery flows are highly non-linear and coupled; thus linear superposition of profile, secondary flow, shock and tip leakage losses gives rise errors.

First of all, the pitch of a section is calculated at the leading edge radial position (relative to machine axis) while solidity is calculated at mid-chord radial position. The second enhancement is that, if overturning occurs (roots of fans), exit metal angle is taken as zero instead of negative value. This is due to the assumption that incidence is mostly affected by leading edge region rather than the exit overturning region. Moreover, the correlations are valid for cases without overturning. In this regard, authors experienced better match in NASA 2-stage fan test case. The third enhancement is that, the exponent ' 2 ' in equation (17) is made ' 1.6 ' to better match test cases. The fourth enhancement is that suction surface PrandtlMeyer expansion is reduced relative to a circular-arc expansion. The circular-arc expansion (for given inlet and outlet angles) is multiplied by 0.95 for inlet relative Mach number smaller than 1.25, multiplied by 0.45 for inlet relative Mach number bigger than 1.40 and a linear distribution is assumed in between these two Mach numbers. The fifth enhancement is related to deviation angle, which is reduced by $1^{\circ}$ relative to predicted values. Moreover, for rotors, an additional $2 \circ$ tip deviation is added, which diminishes at $90 \%$ span. With those modifications, the authors experienced better match with test cases. ${ }^{23}$

\section{Optimization with genetic algorithm}

The bypass fan system under consideration has an outer diameter of $1.5 \mathrm{~m}$. There are 23 fan rotor blades (2.63 hub solidity, 1.28 tip solidity) with average hub-to-tip radius ratio of $0.344,60$ bypass $\mathrm{OGV}$ vanes (2.11 hub solidity, 1.34 tip solidity) and 100 core IGV vanes (1.93 hub solidity, 2.04 tip solidity). Corrected rotor rotation speed is $5700 \mathrm{r} / \mathrm{min}$, corrected mass flow rate is $300 \mathrm{~kg} / \mathrm{s}$. Corrected tip rotation speed is approximately $445 \mathrm{~m} / \mathrm{s}$ and tip inlet relative Mach number is around 1.4. Meridional view of the geometry is shown in Figures 3, 9 and 10.

The problem tackled has two objectives at a time, where maximizing efficiency and total pressure uniformity at station B (or to minimize standard deviation of total pressure distribution) are both simultaneously targeted, by changing the geometry of the flow domain as seen in Figure 3. In order to achieve these two separate objectives, a so called pseudo objective function is defined as in equation (21). Optimization task performed considers

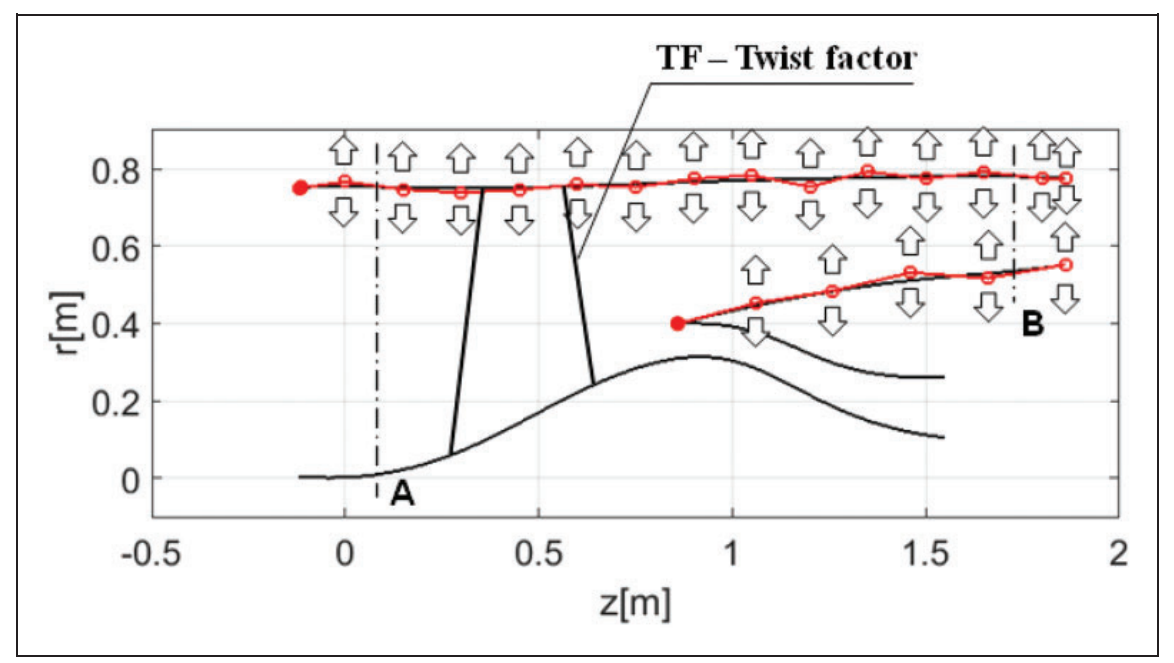

Figure 3. Graphical illustration of the optimization problem and the design variables. 
aerodynamic aspects only, whereas aeromechanical and stress issues are not taken into consideration.

The graphical representation of the problem with design variables is given in Figure 3, where design variables for the problem are:

Tangential velocity $\left(V_{\theta}\right)$ distribution at the rotor discharge: $V_{\theta}$ distribution at the exit of the fan rotor blade is parameterized via twist factor (TF) as formulized in equation (18). Since the mean-line tangential velocity at the rotor discharge $\left(V_{-M L}\right)$ and mean-line radius $\left(r_{M L}\right)$ are known (through a mean-line analysis), value of $V$ at any radial location can be easily found by equation (18). The tangential velocity at the exit station of the rotor directly defines the enthalpy rise that the fan subjects to air passing through blades, due to the well-known Euler's turbomachinery equation, which is given in equation (19). As explained previously, prescribing a tangential velocity distribution directly defines the design criteria. Assigning TF properly will definitely provide a more efficient blade geometry, whereas a bad decision on TF will result in catastrophic failures on the engine's performance.

$$
\begin{aligned}
& V_{\theta}=V_{\theta-M L}\left(\frac{r_{M L}}{r}\right)^{T F} \\
& \Delta H=N r\left(V_{\theta, 2}-V_{\theta, 1}\right)
\end{aligned}
$$

Flow-path of the bypass region: Flow-path's general shape acts either as a nozzle or diffuser, but also the end-wall contour plays a direct role on local acceleration or deceleration of the fluid. Any steep increase or decrease in the shape of the contour may cause undesired separation zones or over-accelerating regions in the flow-field. For this purpose, flow-path geometry is decided to be perturbed. The parameterization of the geometry is conducted using Bézier curves, where the geometry comprises 19 control points as seen in Figure 3.The core region of the engine is kept unchanged in this study.

With the twist factor and the control points of the Bézier curves, the optimization task is performed using 20 design variables.

\section{Geometric and aerodynamic constraints}

Geometric parameters are constrained with the limits demanded by the mechanical design team. The constraint on twist factor (TF), $0.65<T F<0.90$, is posed in order to keep the spanwise blade loading in a reasonable band. In case the blade is overloaded at hub or tip, an undesired flow separation may occur, which may not be estimated in through-flow approximation.

In addition to the constraints concerning design variables, following constraints are decided to be respected.

Diffusion factor provides a measure for the amount of deceleration or diffusion of the surface velocity which eventually determines the wake momentum thickness in 2D cascades. There are two diffusion factors in the literature, diffusion factor and equivalent diffusion factor $\left(D_{e q}\right)$. The latter is implemented within the SLC methodology, which is formulized in equation (20)

$$
D_{e q}=\frac{W_{\max }}{W_{2}}
$$

where $W_{2}$ is station outlet relative velocity and $W_{\max }$ is the maximum relative velocity on the cascade suction side, estimated for a well-designed profile from the comprehensive Koch and Smith correlations. ${ }^{23,32}$

The higher the diffusion factor, the more fan pressurizes the fluid. In other words, the pressure may rise too much in the flow direction; and may go beyond $D_{e q}$ of 2 , hence abrupt increase in losses is observed, ${ }^{23}$ in which case the fluid will no longer follow the blade but separate from the suction surface. Based on these considerations, diffusion factor has to be limited and any design violating the constraint $D_{e q}<2$ should be directly eliminated.

Minimum velocity at the endwalls should be respected as a constraint, where any design violating this constraint will be vulnerable to separation, due to the presence of excessive adverse pressure gradient. This constraint is mathematically expressed in equation (23).

Constraint on maximum meridional velocity gradient also aims to limit the steep diffusion that may occur at the fan. The separation phenomena, which is one of the main loss mechanisms, is tried to be controlled by penalizing the designs, where spatial gradient of the meridional velocity in meridional direction exceeds the value given in equation (24).

Maximum curvature at the endwalls constraint should be respected, where excessive values of curvature $\left(K_{m}\right)$ will cause over acceleration of the fluid at the endwalls, leading to performance losses in the mentioned region. The value for the constraint is decided as given in equation (25).

Total-to-total pressure ratio of the fan is posed as an equality constraint. However, having an equality constraint is computationally more expensive than that of the inequality constraints. Therefore, this equality constraint is converted into an inequality constraint, as $\mathrm{PR}_{\text {min }}<P R<\mathrm{PR}_{\max }$. This way, by keeping the $\mathrm{PR}_{\text {min }}$ and $\mathrm{PR}_{\max }$ close to each other, the equality constraint is virtually obtained. The bounds for this constraint are given in equation (26).

Bypass ratio: The ratio of the mass flow rate bypassing the core and the mass flow rate passing through the core, namely by-pass ratio (BPR), should meet the design constraints. The acceptable range for BPR is given in equation (27). The objectives and constraints explained above problem are summerized in Table 2. 
Table 2. Optimization problem: Objectives and constraints.

\begin{tabular}{lll}
\hline Minimize & $\mathrm{w}_{\mathrm{I}} \times(\mathrm{I} / \eta)+\mathrm{w}_{2} \times \operatorname{std}\left(\overline{P_{\text {out }}^{0}}\right)$ & $(2 \mathrm{I})$ \\
\hline Subject to & $D_{\text {eq }}<2$ & $(22)$ \\
& $V_{\text {hub }}>60 \mathrm{~m} / \mathrm{s}, V_{\text {shroud }}>60 \mathrm{~m} / \mathrm{s}$ & $(23)$ \\
& {$\left[\frac{\partial V_{m}}{\partial m}\right]_{\max }<1.5 \times\left[\frac{\partial V_{m}}{\partial m}\right]_{\text {max }, \text { init }}$} & $(24)$ \\
& $K_{\text {m,ew }}<1.2 \times\left[\max \left(K_{m, \text { ew }}\right)\right]_{\text {init }}$ & $(25)$ \\
& $I .60<P R<1.63$ & $(26)$ \\
& $6.1<B P R<7.46$ & $(27)$ \\
where & $\vec{x}=\left\{R_{1} R_{2} \ldots R_{18} R_{19} T F\right\}^{T}$ \\
\hline
\end{tabular}

PR: total pressure ratio; BPR: by-pass ratio; TF: twist factor.

\section{Optimizer configurations}

Genetic algorithm used in this study is of a conventional one that works with binary representation. Initial population consists of randomly generated 44 individuals. Tournament method is used for the purpose of reproduction. The off-springs are mutated with a probability of $1 \%$. The optimization problem has two objectives (see equation (21)), where each objective is weighted with different weight factor combinations as it will be explained in next sections. The optimization is stopped providing that the optimizer reached a certain number of generations that is set by the designer.

New designs found by genetic algorithm are checked for constraint violations indirectly. This is possible by penalizing the designs that violate the constraints using equations (29) and (30). $G_{j}$ takes the value of ZERO once the constraints are satisfied, that is $g_{j}(\vec{x}) \leqslant 0$; otherwise, square of the constraint function is added as penalty function to the pseudo objective function. $\boldsymbol{r}_{\boldsymbol{k}}$ is the penalty multiplier, and it is increased in successive iterations.

$$
\begin{aligned}
\varphi_{i} & =\varphi\left(\vec{X}, r_{k}\right)=f_{i}(\vec{x})+r_{k} \sum_{j=1}^{m} G_{j}\left[g_{j}(\vec{X})\right] \\
G_{j} & =\left\{\max \left[0, g_{j}(\vec{X})\right]\right\}^{2}
\end{aligned}
$$

Each optimization run is stopped at the end of 40 generations. This corresponds to $\sim 200 \mathrm{~h}$ for each optimization task with different weight factor combinations. In several cases, no objective function improvement may be observed between successive generations. This may mean that the algorithm is trapped into a local minimum. However, the mutation operator helps the algorithm in stepping out the local minimum trap and provides a possibility for obtaining better results in following generations.
Table 3. Optimization results for different weight factor combinations.

\begin{tabular}{llll}
\hline$w_{\text {। }}($ for $\eta)$ & $\begin{array}{l}w_{2} \\
\left(\text { for } \operatorname{Std}\left(\overline{P_{B}^{0}}\right)\right)\end{array}$ & $\begin{array}{l}\text { Improvement } \\
\text { for } \eta\end{array}$ & $\begin{array}{l}\% \operatorname{Improvement} \\
\text { for } \operatorname{Std}\left(\overline{P_{B}^{0}}\right)\end{array}$ \\
\hline 0 & I & NA & 27.54 \\
0.1 & 0.9 & -0.1 & 23.44 \\
0.3 & 0.7 & -0.6 & 20.17 \\
0.5 & 0.5 & -0.2 & 20.71 \\
0.7 & 0.3 & 0.4 & 23.08 \\
0.9 & 0.1 & 0.7 & 22.25 \\
I & 0 & 1.5 & NA \\
\hline
\end{tabular}

NA: Not applicable.

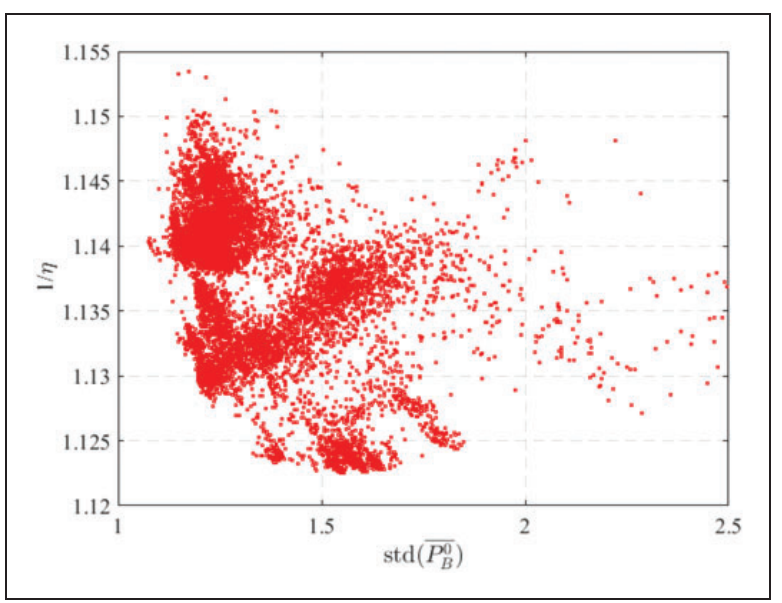

Figure 4. Pareto front obtained with different weight combinations.

\section{Optimization results}

Several optimization tasks are performed using the weight factor combinations tabulated in Table 3 . The same table includes the objective function values and corresponding improvement ratings. Improvement ratings are calculated with reference to the initial family's fittest member's objective function values. Pareto front obtained for various optimization tasks using different weight factor combinations is illustrated in Figure 4.

It is observed that genetic algorithm provided significant improvement in the objective function with respect to the objective function value of the best (or fittest) member of the initial family, as seen in Table 3. The improvement observed in standard deviation of total pressure distribution is remarkably higher than the improvement observed in efficiency. Moreover, in cases where the weight factor for efficiency is smaller than that of the standard deviation of total pressure distribution, the algorithm provides less efficient designs. It is the authors' experience that the total pressure distribution is sensitive to the geometric changes of flow-path and blade's tangential velocity 


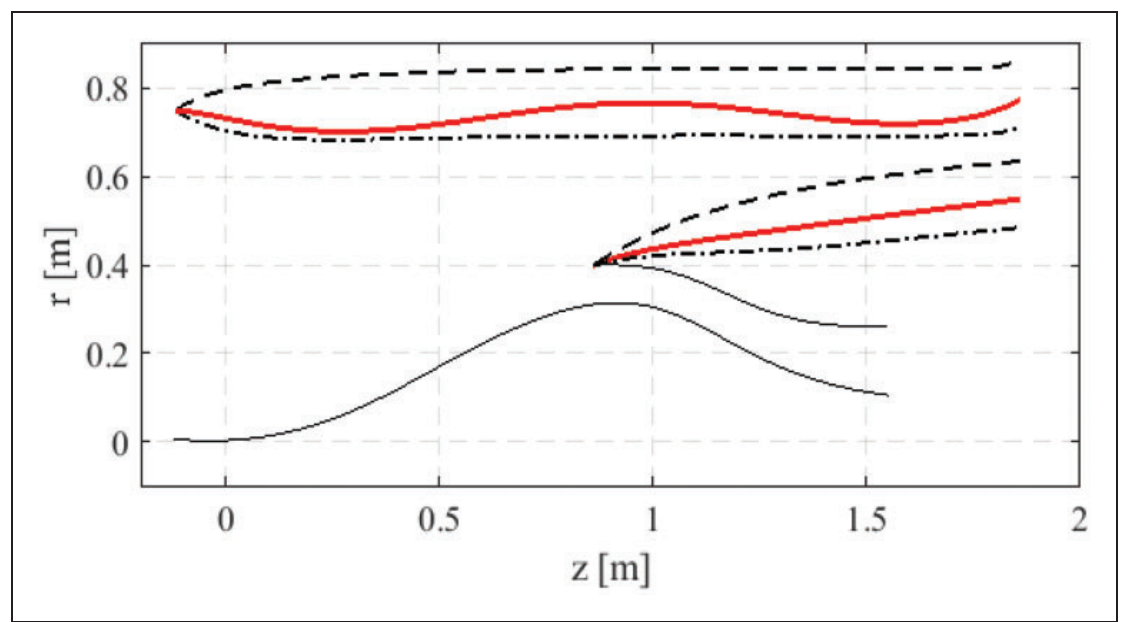

Figure 5. Upper (dashed lines) and lower (dashed and dotted lines) geometric bounds and the optimum geometry (solid thick red lines).

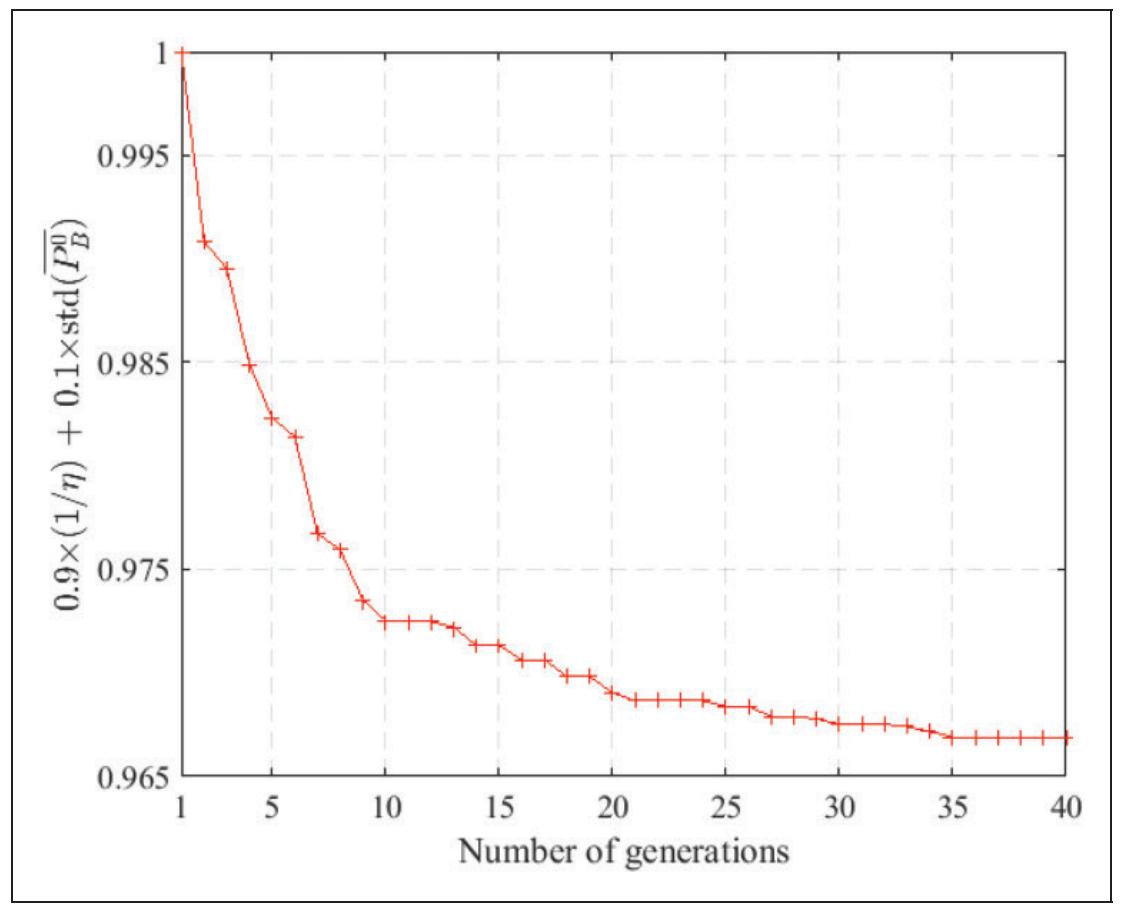

Figure 6. Normalized objective function values at successive generations.

distribution. On the other hand, for the cases where weight factor for efficiency is higher, increase in efficiency values is observed, as well as the other objective function. It should be stated that, although the efficiency improvements are in the order of $0.4-1.5 \%$, the improvement is still important, since such increase will directly decrease the specific fuel consumption of the engine and provide significant economic benefit.

Optimum flow path is plotted in Figure 5 together with geometric lower and upper bounds. Weight combination is picked as $w_{1}=0.9$ and $w_{2}=0.1$ in the following test for detailed representation.
Normalized objective function values at successive iterations are graphed in Figure 6. Constraint values tabulated in Table 4 states that in the final geometry, the limitations are respected and all constraints are satisfied.

Figures 7 and 8 depict the initial best (baselineinitial family's best member) and optimized total pressure and efficiency spanwise distributions at fan outlet, respectively. The discontinuity observed for the span value of $\sim 0.4$ at Figure 7 is an indication that the flow regime goes in to a highly compressible regime in this region, where the correlations used for loss estimation change. The enhancement in efficiency 
Table 4. Through-flow optimization: constraint values.

\begin{tabular}{lll}
\hline & Constraint value & Constraint limit \\
\hline Equivalent DF & 1.604 & $<2$ \\
$\mathrm{~V}_{\text {hub }}, \mathrm{V}_{\text {shroud }}$ & $107.5 \mathrm{~m} / \mathrm{s}$ & $>60 \mathrm{~m} / \mathrm{s}$ \\
$\mathrm{R}_{\mathrm{c}, \text { endwalls }}$ & $1.17 \mathrm{~m}$ & $<1.70 \mathrm{~m}$ \\
$\left.\frac{\partial V_{m}}{\partial m}\right]_{\text {bypass }}$ & $1099 \mathrm{I} / \mathrm{s}$ & $\left.<1.5 \max \left(\frac{\partial V_{m}}{\partial m}\right]_{\text {bypass }}\right)_{\text {initial }}$ \\
PR & 1.612 & $1.60<P R<1.63$ \\
BPR & 6.96 & $6.23<B P R<7.54$ \\
\hline
\end{tabular}

DF: diffusion factor; PR: total pressure ratio; BPR: by-pass ratio; TF: twist factor.

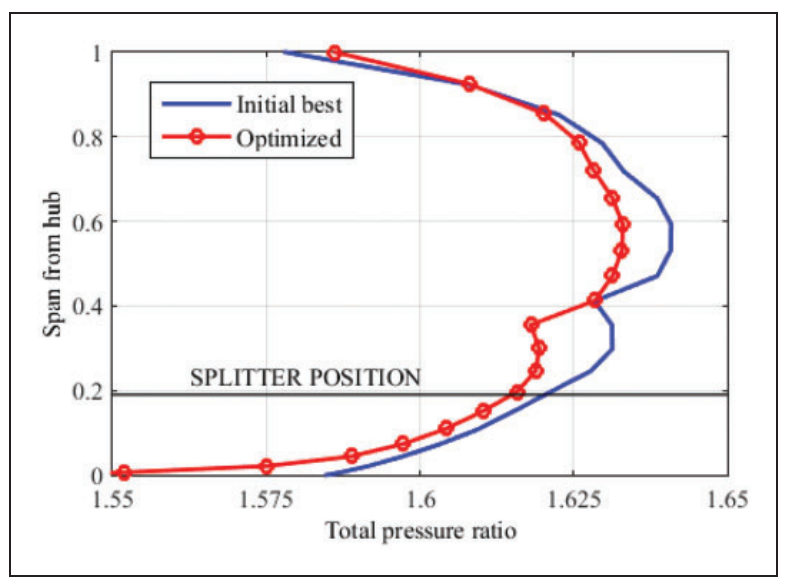

Figure 7. Total-to-total pressure ratio distributions at fan outlet.

can clearly be observed in Figure 8 . However, there is a drop at the fan root ( $<5 \%$ span). To better understand the reason of this, fan rotor equivalent diffusion factor $\left(\mathrm{DF}=\mathrm{W}_{\max } / \mathrm{W}_{\text {exit }}\right.$ ) spanwise distributions are plotted at Figure 9. An unexpected slight increase at the root region $(<5 \%$ span $)$ is evident. The actual reason of this is detected to be the throat margin (excess throat area over the actual flow-normal area for the investigated fan operating point), whose radial distribution is plotted in Figure 10. At the root, the streamtubes are choked and throat margins are negative. Normally, the given minimum loss ( optimum) correlations (equations (2) to (12)) adjust metal angles such that flow passes without choke. In the current fan root, this failed. Considering very high solidity at the root (2.63), this maybe something expected. Due to the parametrization of twist factor, a very detailed rotor exit swirl velocity distribution ultimately best for each region may not be possible and the root may be negatively affected. A slight post-optimization modification to the exit swirl velocity at this region $(<5 \%$ span) may be necessary. Fan root flowpath can also be made concave near the leading edge to increase throat area there, as done typically. However, the authors avoided those typical post-optimization adjustments and intent only presenting the automatic optimization results.

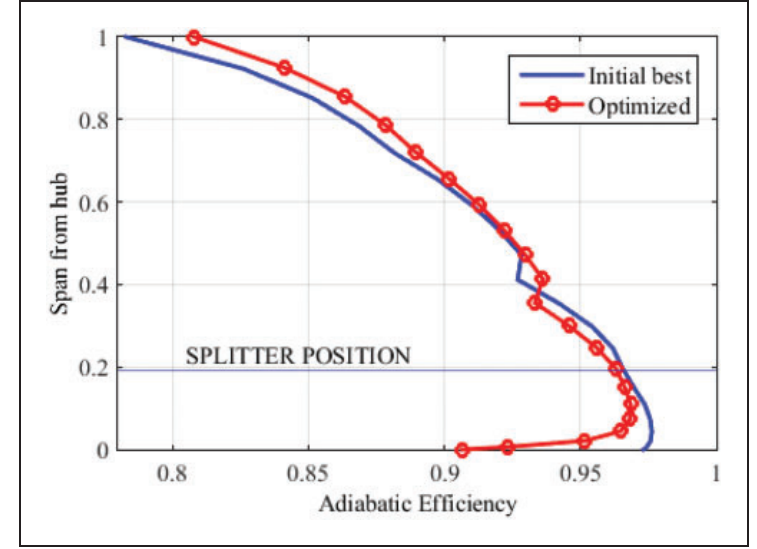

Figure 8. Isentropic total-to-total fan efficiency distributions at fan outlet.

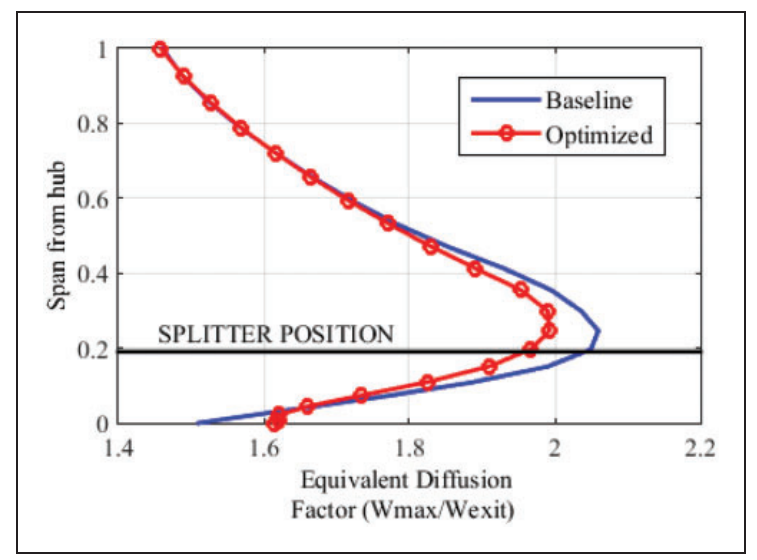

Figure 9. Estimated fan rotor equivalent diffusion factors $\left(W_{\text {max }} / W_{\text {exit }}\right)$ for the initial best and optimized cases.

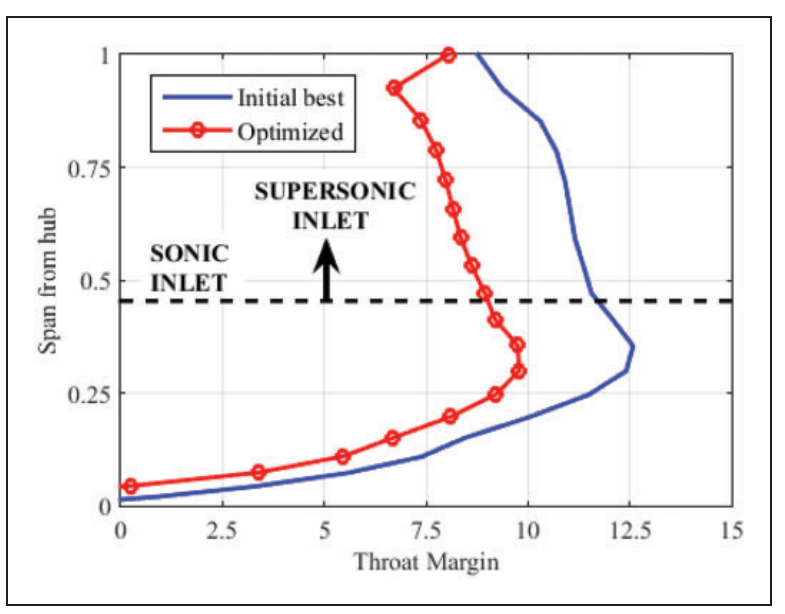

Figure 10. Estimated fan rotor throat margins for the initial best and optimized cases. 


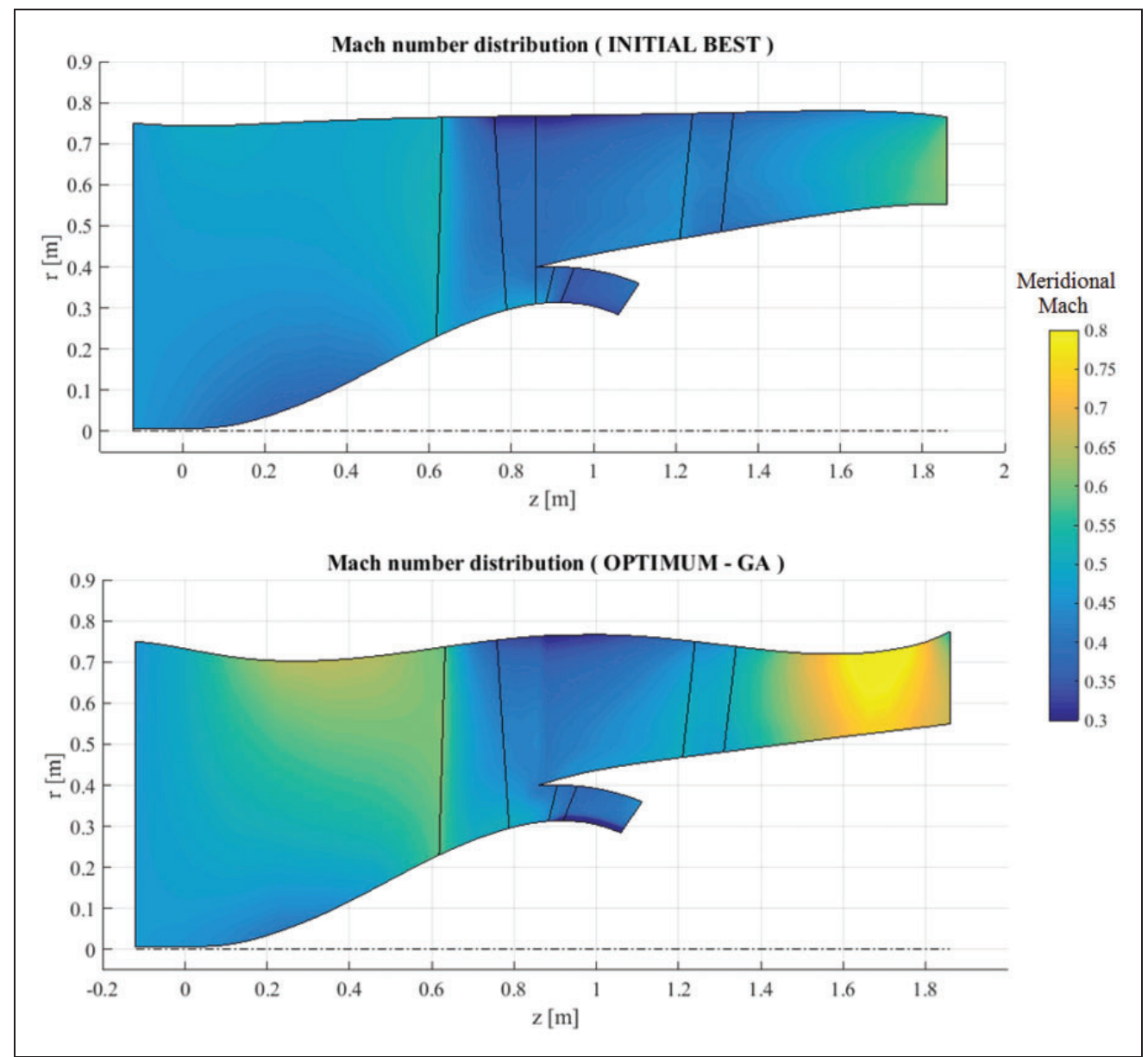

Figure II. Mach number distribution for selected individuals.

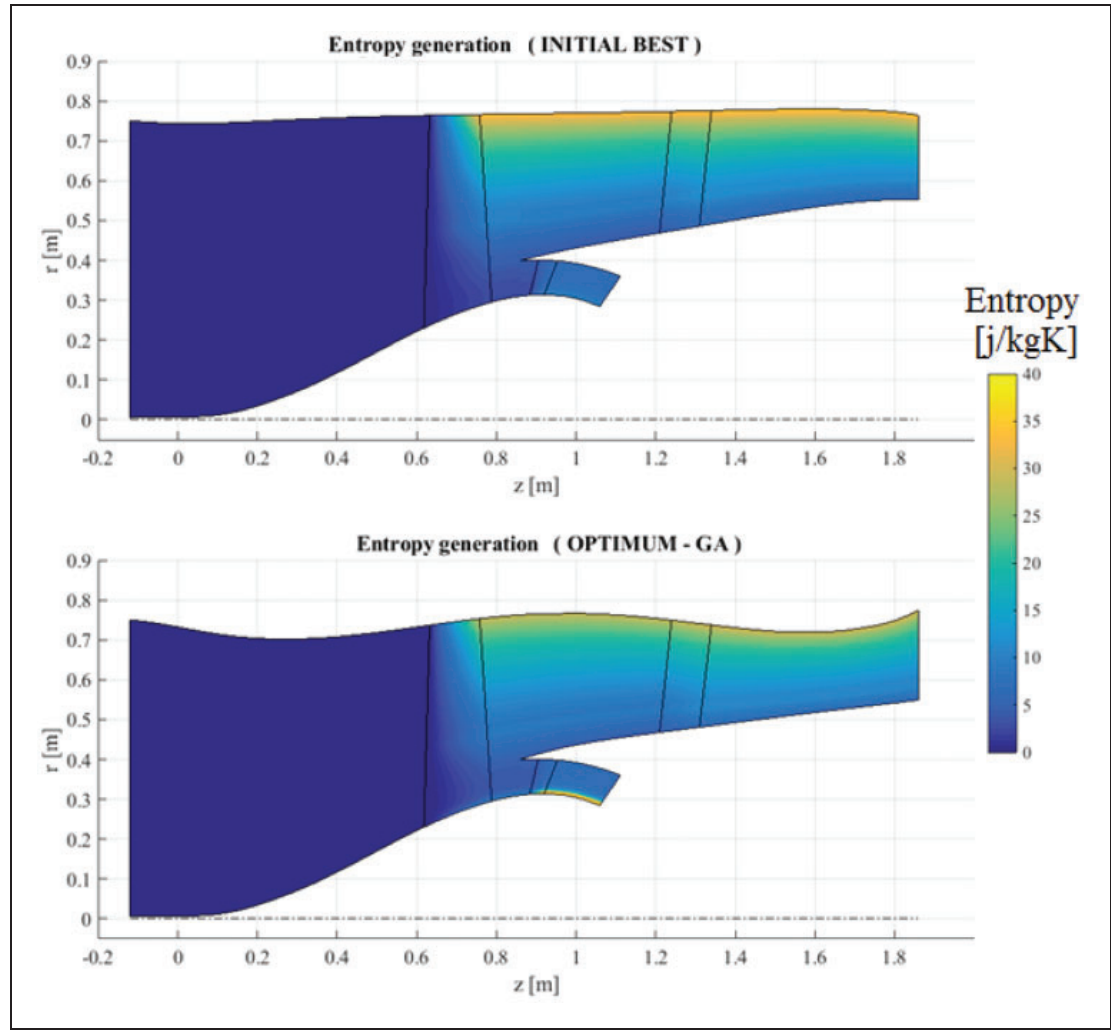

Figure 12. Entropy generation distribution for selected individuals. 
The plots of the Mach number and entropy generation contours of optimum and initial best geometries are provided in Figures 11 and 12. It can be deduced from the figures that for the given design limitations, optimum geometry is obtained for the case where bypass area is comparatively small, resulting in higher outlet Mach number values and smaller by-pass ratios. The BPR change is assessed to be within the specified tolerance and its impact in the cycle has not been performed. But for this specific case, it probably will have a negative impact on the propulsive efficiency. BPR tolerance margins can even be made narrower for this case, or better, the splitter leading edge position can very slightly be modified manually after the optimization process to adjust to the same bypass ratio, without any significant effect on the turbomachinery performance.

\section{Conclusions}

Reduced-order models of turbomachinery aerodynamics are the backbone of design, since great majority of design decisions are made during oneor two-dimensional models. In this regard, twodimensional through-flow solvers are probably the most valuable tools for turbomachinery designers due to having great flexibility and ability to work in inverse design, off-design analysis and test data processing modes. Automatic optimization with advanced optimization algorithms, on the other hand, is incorporated in all fields of design industry and these are the backbone of any modern-era design. In this study, an in-house genetic algorithm optimizer is incorporated with a previously developed in-house through-flow solver. The study considers a unified through-flow model of the fan module such that the flow splitter and two downstream ducts are considered in a realistic and coupled way. Even if the simplified cases of lower-speed and single-stream compressors and turbines are considered for throughflow-based automatic optimization in the literature, the more challenging case of unified high-speed bypass fan design problem, to the authors' best knowledge, is an untouched subject in the field. Geared towards increasing accuracy of through-flow design environment of the bypass fans by the inclusion of the splitter and two downstream ducts, and therefore taking into account the true fan-duct coupling, the performance of an automatic optimizer in such a challenging and previously untouched model is presented in this study.

The coupling of both in-house codes results insignificant improvement in objective function is achieved without violating the constraints.

\section{Declaration of Conflicting Interests}

The author(s) declared no potential conflicts of interest with respect to the research, authorship, and/or publication of this article.

\section{Funding}

The author(s) disclosed receipt of the following financial support for the research, authorship, and/or publication of this article: This work was supported by the Tusaş Engine Industries.

\section{References}

1. Joly M, Verstraete T and Paniagua G. Full design of a highly loaded fan by multi-objective optimization of through-flow and high-fidelity aero-mechanical performances. In: ASME Turbo Expo. Denmark: Copenhagen, 2012.

2. Shahpar S. A comparative study of optimisation methods or aerodynamic design of turbomachinery blades. In: ASME Turbo Expo. Germany: Munich, 2000.

3. Rao S. Engineering optimization, theory and practice. 3rd ed. New York: John Wiley and Sons, Inc., 1996.

4. Giles $M$ and Pierce N. An introduction to the adjoint approach to design. Flow Turbul Combust 2000; 65: 393-415.

5. Shahpar S. Optimisation strategies used in turbomachinery design from an industrial perspective. In: von Karman Institute for fluid dynamics lecture series. Belgium: Rhode St. Genése, 2012.

6. Papadimitriou DI, Zymaris AS and Giannakoglou KC. Discrete and continuous adjoint formulations for turbomachinery applications. In: Evolutionary and deterministic methods for design, optimization and control with applications to industrial and societal problems, Germany: Munich, 2005.

7. Wang DX and He L. Adjoint aerodynamic design optimization for blades in multistage turbomachines - part I: methodology and verification. J Turbomach 2010; 132: 1-14.

8. Massardo A and Satta A. Axial flow compressor design optimization: part I - pitchline analysis and multivariable objective function influence. J Turbomach 1990; 112: 399-404.

9. Massardo A, Satta A and Marini M. Axial flow compressor optimization: part II - throughflow analysis. $J$ Turbomach 1990; 112: 405-410.

10. Burguburu S. Numerical optimization of turbomachinery bladings. J Turbomach 2004; 126: 91-100.

11. Andersson J. Multiobjective optimization in engineering design - applications to fluid power systems. $\mathrm{PhD}$ Thesis, Linköpings Universitet - Institute of Technology, Linköping, Sweden, 2001.

12. Pierret S. Designing turbomachinery blades by means of the function approximation concept based on artificial neural network, genetic algorithm, and the Navier-Stokes equations. PhD Thesis, von Karman Institute for Fluid Dynamics, Rhode St. Genése, Belgium, 1999.

13. Van den Braembussche R. Numerical optimization for advanced turbomachinery. In: D Thévenin and G Janiga (eds) Optimization and computational fluid dynamics. Berlin: Springer, 2008, pp.147-188.

14. Pierret S and Hirsch C. An integrated optimization system for turbomachinery blade shape design. In: RTO AVT symposium on "reduction of military vehicle acquisition time and cost through advanced modelling and virtual simulation”. France: Paris, 2002. 
15. Pierret $\mathrm{S}$ and Van den Braembussche $\mathrm{R}$ Turbomachinery blade design using a Navier-Stokes solver and artificial neural network. $J$ Turbomach 1999; 121: 326-332.

16. Ahn $\mathrm{C}$ and Kim K. Aerodynamic design optimization of an axial flow compressor rotor. In: ASME Turbo Expo. Amsterdam, The Netherlands, 2002.

17. Giannakoglou K. Designing turbomachinery blades using evolutionary methods. In: ASME Turbo Expo. USA: Indianapolis, 1999.

18. Lian Y and Liou M. Multi-objective optimization of transonic compressor blade using evolutionary algorithm. J Propul Power 2005; 21: 979-987.

19. Okui H, Verstraete T, Van den Braembussche R, et al. Three dimensional design and optimization of a transonic rotor in axial flow compressors. In: ASME Turbo Expo. Canada: Vancouver, British Columbia, 2011.

20. Petrovic M, Dulikravich G and Martin T. Optimization of multistage turbines using a throughflow code. Proc IMechE Part G: Journal of Aerospace Engineering 2001; 215: 559-569.

21. Park K, Turner M, Siddappaji K, et al. Optimization of a 3-stage booster. Part I - the axissymmetric multi-disciplinary optimization approach to compressor design. In: ASME Turbo Expo. Canada: Vancouver, British Columbia, 2011.

22. Joly M, Verstraete T and Paniagua G. Full design of a highly loaded fan by multi-objective optimization of through-flow and high-fidelity aero-mechanical performance. In: Proceedings of ASME Turbo Expo 2012, Copenhagen, Denmark, 2012.

23. Acarer S and Özkol Ü. An extension of the streamline curvature through-flow design method for bypass fans of turbofan engines. Proc IMechE Part G: Journal of Aerospace Engineering 2017; 231: 240-253.

24. Acarer $\mathrm{S}$ and Özkol Ü. Off-design analysis of transonic bypass fan systems using streamline curvature throughflow method. Int $J$ Turbo Jet-Engines 2017. DOI: 10.1515/tjj-2016-0083.

25. Kor O. Aerodynamic optimization of a transonic aero engine fan module. PhD Thesis, İzmir Institute of Technology, İzmir, Turkey, 2016.

26. Aungier R. Axial-flow compressors. New York: ASME Press, 2003.

27. Novak RA. Streamline curvature computing procedures for fluid flow problems. Trans ASME $J$ Eng Power 1967; 89: 478-490.

28. Lieblein S. Incidence and deviation-angle correlations for compressor cascades. Trans ASME J Basic Eng 1960; 82: 575-584.

29. Bullock R and Johnsen I. Aerodynamic design of axial flow compressors. NASA SP36, 1965.

30. Kleppler J. Technique to predict stage-by-stage, prestall compressor performance characteristics using a streamline curvature code with loss and deviation correlations. MSc Thesis, University of Tennessee, Tennessee, USA, 1998.

31. Boyer KM. An improved streamline curvature approach for off-design analysis of transonic compression systems. PhD Thesis, Virginia Polytechhnic Institute and State University, Virginia, USA, 2001.

32. Koch $\mathrm{C}$ and Smith L. Loss sources and magnitudes in axial-flow compressors. J Eng Power 1976; 98: 411-424.

33. Miller G, Lewis G Jr and Hartmann M. Shock losses in transonic compressor blade rows. J Eng Power 1961; 83: 235-241.

34. Wennerstrom AJ. Design of highly loaded axial-flow fans or compressors. White River Junction, VT: Concepts Eti, 2001.

35. Pachidis V. Gas turbine advanced performance simulation. PhD Thesis, Cranfield University, London, UK, 2006.

36. Çetin M, Üçer AŞ, Hirsch C, et al. Application of modified loss and deviation correlations to transonic axial compressors. AGARD R745, 1987.

37. Creveling H. Axial-flow compressor computer program for calculating off-design performance. NASA CR72472, 1968.

38. Urasek D, Gorrell W and Cunnan W. Performance of two-stage fan having low-aspect-ratio first stage rotor blading. NASA TP-1493, 1979.

39. Sullivan T, Silverman I and Little D. Single stage, low noise advanced technology fan, volume 4: fan aerodynamics. NASA CR-134892, 1977.

\section{Notation}

$a / c$

b

$c$

$C_{1}, C_{2}$

$D_{e q}$

$f$

$F$

$F_{q}$

g, G

H

$i^{*}$

$i_{c}$

$i_{m}$

$i_{s}$

$K_{1}, K_{2}$

$K_{m}$

$K_{s h}$

$m$

$M_{1}^{\prime}$

$N$

$o$

$P^{O}$

$q$

$r$

$r_{k}$

$R_{c}$

$R_{s}$

$s$

$t$ maximum camber point location maximum distance between camberline and chordline

chord

calibration coefficients

equivalent diffusion factor

individual objective function

population objective function

local blade force along quasi-

orthogonal

constraint function

total enthalpy

reference minimum-loss incidence angle reference choke incidence (loss two times the design loss)

Mach-corrected minimum-loss incidence angle

reference stall incidence (loss two times the design loss)

inlet and outlet metal angles

local streamline curvature

profile shape factor

meridional direction

inlet relative Mach number

angular velocity of the rotor

geometric throat opening

stagnation (total) pressure

local directional on quasi-orthogonals

radius

penalty multiplier

reference angular range between choke and design point

reference angular range between stall and design point

entropy

pitch 


$\begin{array}{llll}t h & \text { maximum profile thickness } & \sigma & \text { solidity } \\ T & \text { temperature } & \phi & \text { streamline slope } \\ V_{m} & \text { meridional velocity } & \varphi & \text { penalized objective function } \\ w_{1}, w_{2} & \text { optimization weights } & \psi & \text { modified stagger parameter } \\ W & \text { blade profile relative velocity }\left(W_{1}-\right. & \omega^{*} & \text { reference friction loss coefficient } \\ & \text { inlet; } W_{2}-\text { outlet; } W_{\max }-\text { maximum at } & \omega_{\text {friction }} & \text { Mach-corrected friction loss coefficient } \\ & \text { suction side) } & \omega_{\text {shock }} & \text { shock loss coefficient }\end{array}$

$\beta \quad$ flow angle ( $\beta_{1}-$ inlet; $\beta_{2}-$ outlet $)$

$\gamma \quad$ stagger

$\delta^{*} \quad$ deviation corresponding to reference

minimum-loss incidence angle

local angle between streamline true

orthogonals and quasi-orthogonals

adiabatic efficiency

camber angle

$\rho \quad$ air density

\section{Subscripts}

ew

endwall

init initial value

n iteration number

$\theta \quad$ tangential direction 